\title{
Highly stretchable and tough hydrogels
}

\author{
Jeong-Yun Sun ${ }^{1,2}$, Xuanhe Zhao ${ }^{3}$, Widusha R. K. Illeperuma ${ }^{1}$, Ovijit Chaudhuri ${ }^{1}$, Kyu Hwan \\ $\mathrm{Oh}^{2}$, David J. Mooney ${ }^{1,4}$, Joost J. Vlassak ${ }^{1}$, and Zhigang Suo ${ }^{1,5}$ \\ ${ }^{1}$ School of Engineering and Applied Sciences, Harvard University, Cambridge, MA 02138, USA \\ ${ }^{2}$ Department of Material Science and Engineering, Seoul National University, Seoul 151-742, \\ South Korea \\ ${ }^{3}$ Department of mechanical Engineering and Materials Science, Duke University, Durham, NC \\ 27708, USA \\ ${ }^{4}$ Wyss Institute for Biologically Inspired Engineering, Harvard University, Cambridge MA 02138 , \\ USA \\ ${ }^{5}$ Kavli Institute for Bionano Science and Technology, Harvard University, Cambridge MA 02138, \\ USA
}

\begin{abstract}
Hydrogels are used as scaffolds for tissue engineering [1], vehicles for drug delivery [2], actuators for optics and fluidics [3], and model extracellular matrices for biological studies [4]. The scope of applications, however, is often severely limited by the mechanical behavior of hydrogels [5]. Most hydrogels do not exhibit high stretchability. For example, an alginate hydrogel ruptures when stretched to about 1.2 times its original length. Some synthetic elastic hydrogels $[6,7]$ have achieved stretches in the range of 10-20, but elastic gels are known to reduce achievable stretches markedly when samples contain notches. Most hydrogels are brittle, having fracture energy on the order of $10 \mathrm{~J} / \mathrm{m}^{2}$ [8]. By comparison, the fracture energy is $\sim 1000 \mathrm{~J} / \mathrm{m}^{2}$ for cartilage [9] and $\sim 10,000 \mathrm{~J} / \mathrm{m}^{2}$ for natural rubbers [10]. Intense efforts are devoted to synthesizing hydrogels of enhanced mechanical properties [11-18]; certain synthetic gels have reached fracture energy of 100-1000 J/m² $[11,14,17]$. Despite the exciting achievements, much of the property space of hydrogels remains uncharted. Here we report hydrogels made of polymers forming networks via ionic and covalent crosslinks. Although such a gel contains $~ 90 \%$ water, it can be stretched beyond 20 times its initial length, and has fracture energy of $\sim 9000 \mathrm{~J} / \mathrm{m}^{2}$. Even for samples containing notches, a stretch of 17 is demonstrated. The toughness is attributed to the synergy of two mechanisms: crack bridging by the network of covalent crosslinks, and hysteresis by unzipping the network of ionic crosslinks. Furthermore, the network of covalent crosslinks preserves the memory of the initial state, so that much of the large deformation is removed upon unloading. The unzipped ionic crosslinks cause internal damage, which heals by re-zipping. These gels may serve as model systems to explore
\end{abstract}

\footnotetext{
Correspondence and requests for materials should be addressed to Z.G.S. (suo@seas.harvard.edu).

Supplementary Information is linked to the online version of the paper at www.nature.com/nature.

Author Contributions J.Y.S., X.H.Z., W.R.K.I., D.J.M., J.J.V. and Z.G.S. designed the study and interpreted the results. X.H.Z. developed the protocol for fabrication of the gels and prepared initial samples. J.Y.S. and W.R.K.I. improved the protocol, and performed mechanical tests and recovery tests. J.Y.S. obtained Fourier Transform Infrared spectra and performed thermogravimetric analysis. O.C. and J.Y.S. conducted the experiment with fluorescent alginate and the experiment using the atomic force microscope. K.H.O. contributed to the discussion of results. J.Y.S., W.R.K.I. and Z.G.S. wrote the manuscript. All authors commented on the manuscript.

Reprints and permissions information is available at www.nature.com/reprints.

The authors declare no competing financial interests.
} 
mechanisms of deformation and energy dissipation. Hydrogels with enhanced mechanical properties will expand the scope of their applications.

Certain synthetic hydrogels have achieved exceptional mechanical behavior. A hydrogel containing slide-ring polymers can be stretched more than 10 times its initial length [6]; a tetra-poly (ethylene glycol) gel exhibits strength of $\sim 2.6 \mathrm{MPa}$ [7]. These gels deform elastically. An elastic gel is known to be brittle and notch-sensitive- that is, the high stretchability and strength drop markedly when samples contain notches, or any other features that cause inhomogeneous deformation [19]. A gel can be made tough and notchinsensitive by introducing energy-dissipating mechanisms. For example, fracture energy of $\sim 1000 \mathrm{~J} / \mathrm{m}^{2}$ is achieved with a double-network gel, in which two networks are separately crosslinked by covalent bonds, one network having short chains, and the other having long chains [11]. When the gel is stretched, the short-chain network ruptures and dissipates energy [20]. The rupture of the short-chain network, however, causes permanent damage. After the first loading the gel does not recover from the damage; on subsequent loadings the fracture energy is much reduced [21]. To enable recoverable energy-dissipating mechanisms, several recent works have replaced the sacrificial covalent bonds with noncovalent bonds. In a gel with a copolymer of triblock chains, for example, the end blocks of different chains form glassy domains, and the midblocks of different chains form ionic crosslinks [22]. When the gel is stretched, the glassy domains remain intact, while the ionic crosslinks break and dissipate energy. The ionic crosslinks reform during a period of time after the first loading [22]. Recoverable energy dissipation can also be effected by hydrophobic associations $[17,18]$. When a gel made with hydrophobic bilayers in a hydrophilic polymer network is stretched, the bilayers dissociate and dissipate energy; upon unloading, the bilayers re-assemble, leading to recovery [17]. The existing works, however, have demonstrated fracture energy comparable to, or lower than, that of the double-network gels.

Here we demonstrate extremely stretchable and tough hydrogels by mixing two types of crosslinked polymers: ionically crosslinked alginate and covalently crosslinked polyacrylamide (Fig. 1). An alginate chain consists of mannuronic acid (M unit) and guluronic acid ( $\mathrm{G}$ unit), arranged in blocks rich in $\mathrm{G}$ units, blocks rich in $\mathrm{M}$ units, and blocks of alternating $\mathrm{G}$ and $\mathrm{M}$ units. In an aqueous solution, the $\mathrm{G}$ blocks on different alginate chains form ionic crosslinks through divalent cations (e.g., $\mathrm{Ca}^{2+}$ ), resulting in a network in water-an alginate hydrogel. By contrast, in a polyacrylamide hydrogel, the polyacrylamide chains form a network by covalent crosslinks. Powders of alginate (FMC Biopolymer, LF 20/40) and acrylamide (Sigma, A8887) were dissolved in deionized water. Unless otherwise stated, the water content was fixed at $86 \mathrm{wt} \%$. Ammonium persulfate (AP; Sigma, A9164) was added as a photo initiator for polyacrylamide, and N,N-methylenebisacrylamide (MBAA; Sigma, M7279) was added as the crosslinker for polyacrylamide. After degassing the solution in a vacuum chamber, $\mathrm{N}, \mathrm{N}, \mathrm{N}^{\prime}, \mathrm{N}^{\prime}$-tetramethylethylenediamine (TEMED; Sigma, T7024), 0.0025 the weight of acrylamide, was added as the crosslinking accelerator for polyacrylamide. Calcium sulfate slurry $\left(\mathrm{CaSO}_{4} 2 \mathrm{H}_{2} \mathrm{O}\right.$; Sigma, 31221) was added as the ionic crosslinker for alginate. The solution was poured into a glass mold, $75.0 \times 150.0 \times 3.0$ $\mathrm{mm}^{3}$, covered with a 3-mm thick glass plate. The gel was cured in one step with ultraviolet light (Hoefer, UVC 500) for 1 hour, with $8 \mathrm{~W}$ power and $254 \mathrm{~nm}$ wavelength at $50{ }^{\circ} \mathrm{C}$. The gel was then left in a humid box for 1 day to stabilize the reactions. After curing, the gel was taken out of the humid box, and water on the surfaces of the gel was removed with $\mathrm{N}_{2}$ gas for 1 minute.

The gel was glued to two clamps made of polystyrene, resulting in specimens of $75.0 \times 5.0$ $\times 3.0 \mathrm{~mm}^{3}$. All mechanical tests were performed in air, at room temperature, using a tensile machine (Instron model 3342) with a 500-N load cell. In both loading and unloading, the 
rate of stretch was kept constant at 2 per minute. We stretched an alginate-polyacrylamide hybrid gel over 20 times its original length without rupture (Fig. 2a,b). The hybrid gel was also extremely notch-insensitive. When we cut a notch into the gel (Fig. 2c) and then pulled the gel to a stretch of 17, the notch was dramatically blunted and remained stable (Fig. 2d). At a critical applied stretch, a crack initiated at the front of the notch, and ran rapidly through the entire sample (Supplementary Movie 1). Large, recoverable deformation is demonstrated by dropping a metal ball on a membrane of the gel fixed by circular clamps (Supplementary Movie 2). Upon hitting the membrane, the ball stretched the membrane greatly and then bounced back. The membrane remained intact, vibrated, and recovered its initial flat configuration after the vibration was damped out. A ball of a higher kinetic energy, however, caused the membrane to rupture after large deformation (Supplementary Movie 3).

The extremely stretchable hybrid gels are even more remarkable when compared with their parents: the alginate gel and the polyacrylamide gel (Fig. 3a). The amounts of alginate and acrylamide in the hybrid gels were kept the same as those in the alginate gel and polyacrylamide gel, respectively. When the stretch was small, the elastic modulus of the hybrid gel was $29 \mathrm{kPa}$, which was close to the sum of the elastic modulus of the alginate gel $(17 \mathrm{kPa})$ and that of the polyacrylamide gel $(8 \mathrm{kPa})$. The stress and the stretch at rupture were, respectively, $156 \mathrm{kPa}$ and 23 for the hybrid gel, $3.7 \mathrm{kPa}$ and 1.2 for the alginate gel, and 11 $\mathrm{kPa}$ and 6.6 for the polyacrylamide gel. That is, the properties at rupture of the hybrid gel far exceeded those of either of its parents.

Hybrid gels dissipate energy effectively, as shown by pronounced hysteresis. The area between the loading and unloading curves of a gel gave the energy dissipated per unit volume (Fig. 3b). The alginate gel exhibited pronounced hysteresis and retained significant permanent deformation after unloading. In contrast, the polyacrylamide gel showed negligible hysteresis, and the sample fully recovered its original length after unloading. The hybrid gel also showed pronounced hysteresis, but the permanent deformation after unloading was significantly smaller than that of the alginate gel. The pronounced hysteresis and relatively small permanent deformation of the hybrid gel were further demonstrated by loading several samples to large values of stretch before unloading (Fig. 3c).

After the first loading and unloading, the hybrid gel was much weaker if the second loading was applied immediately, and recovered somewhat if the second loading was applied 1 day later (Fig. 3d and Supplementary Fig. 1). We loaded a sample of the hybrid gel to a stretch of 7, and then unloaded the gel to zero force. The sample was then sealed in a polyethylene bag and submerged in mineral oil to prevent water from evaporation, and stored in a bath of a fixed temperature for a certain period of time. The sample was taken out of the storage and its stress-stretch curve was measured again at room temperature. The internal damage was much better healed by storing the gel at an elevated temperature for some time before reloading (Fig. 3e and Supplementary Fig. 2). After storing at $80^{\circ} \mathrm{C}$ for 1 day, the work on reloading was recovered to $74 \%$ of that of the first loading (Fig. 3f).

Gels of various proportions of alginate and acrylamide were prepared to study why the hybrids were much more stretchable and stronger than either of their parents. When the proportion of acrylamide was increased, the elastic modulus of the hybrid gel was reduced (Fig. 4a). However, the critical stretch at rupture reached the maximum when acrylamide was 89 wt.- $\%$. A similar trend was observed for samples with notches (Fig. 4c). The fracture energy reached a maximum value of $8700 \mathrm{~J} / \mathrm{m}^{2}$ when acrylamide was $86 \mathrm{wt.}$-\% (Fig. 4d). The densities of ionic and covalent crosslinks also strongly affect the mechanical behavior of the hybrid gels (Supplementary Figs. 3 and 4), as well as that of pure alginate gels (Supplementary Fig. 5) and pure polyacrylamide gels (Supplementary Fig. 6). 
On the basis of our experimental findings, we discuss mechanisms of deformation and energy dissipation. When an unnotched hybrid gel is subject to a small stretch, the elastic modulus of the hybrid gel is nearly the sum of that of the alginate gel and that of the polyacrylamide gel. This behavior is further ascertained by viscoelastic moduli determined for the hybrid and pure gels (Supplementary Fig. 7). Thus, in the hybrid gel the alginate and the polyacrylamide chains both bear loads. Moreover, alginate is finely dispersed in the hybrid gel homogeneously, as demonstrated by using fluorescent alginate and by measuring local elastic modulus with atomic force microscopy (Supplementary Fig. 8). The load sharing of the two networks may be achieved by entanglements of the polymers, and by possible covalent crosslinks formed between the amine groups on polyacrylamide chains and the carboxyl groups on alginate chains (Fig. 1, Supplementary Figs. 9 and 10). As the stretch increases, the alginate network unzips progressively [23], while the polyacrylamide network remains intact, so that the hybrid gel exhibits pronounced hysteresis and little permanent deformation. Since only the ionic crosslinks are broken, and the alginate chains themselves remain intact, the ionic crosslinks can reform, leading to the healing of the internal damage.

The giant fracture energy of the hybrid gel is remarkable, considering that its parents-the alginate gel and polyacrylamide gel-have fracture energies in the range of $10-250 \mathrm{~J} / \mathrm{m}^{2}$ (Supplementary Figs. 5 and 6). The relatively low fracture energy of a hydrogel of a single network with covalent crosslinks is understood in terms of the Lake-Thomas model [8]. When the gel contains a notch and is stretched, the deformation is inhomogeneous: the network directly ahead the notch is stretched more than elsewhere (Supplementary Fig. 11). For the notch to turn into a running crack, only the chains directly ahead the notch needs to break. Once a chain breaks, the energy stored in the entire chain is dissipated. In the ionically crosslinked alginate, fracture proceeds by unzipping ionic crosslinks and pulling out chains [24]. After one pair of G blocks unzip, the high stress shifts to the neighboring pair of G blocks and causes them to unzip also (Supplementary Fig. 11). For the notch in the alginate gel to turn into a running crack, only the alginate chains crossing the crack plane need to unzip, leaving the network elsewhere intact. In both polyacrylamide gel and alginate gel, rupture results from localized damage, leading to small fracture energies.

That a tough material can be made of brittle constituents is reminiscent of transformationtoughening ceramics, as well as composites made of ceramic fibers and ceramic matrices. The toughness of the hybrid gel can be understood by adapting a model well studied for toughened ceramics [25] and for gels of double networks of covalent crosslinks [26,27]. When a notched hybrid gel is stretched, the polyacrylamide network bridges the crack and stabilizes deformation, enabling the alginate network to unzip over a large region of the gel (Supplementary Fig. 11). The unzipping of the alginate network, in its turn, reduces the stress concentration of the polyacrylamide network ahead the notch. The model highlights the synergy of the two toughening mechanisms: crack bridging and background hysteresis.

The idea that gels can be toughened by mixing weak and strong bonds has been exploited in several ways, including hydrophobic associations [18], particle filled gels $[7,15]$ and supramolecular chemistry [17,22]. The fracture energy of the alginate-polyacrylamide hybrid gel, however, is much larger than previously reported values of tough synthetic gels $\left(100-1000 \mathrm{~J} / \mathrm{m}^{2}\right)[14,17,20,28]$, a finding which we attribute to how the alginate network unzips. Each alginate chain contains a large number of $\mathrm{G}$ blocks, many of which form ionic crosslinks with $\mathrm{G}$ blocks on other chains when enough $\mathrm{Ca}^{++}$ions are present [1]. When the hybrid gel is stretched, the polyacrylamide network remains intact and stabilizes the deformation, while the alginate network unzips progressively, with closely spaced ionic crosslinks unzipping at a small stretch, followed by more and more widely spaced ionic crosslinks unzipping as the stretch increases. 
Because of the large magnitude of the fracture energy and the pronounced blunting of the notches, we ran a large number of experiments to determine the fracture energy, using three types of specimens, as well as changing the size of the specimens (Supplementary Figs. 1216). The experiments showed that the measured fracture energy is independent of the shape and size of the specimens.

Our data suggest that the fracture energy of hydrogels can be dramatically enhanced by combining weak and strong crosslinks. The combination of relatively high stiffness, high toughness and recoverability of stiffness and toughness, along with an easy method of synthesis, make these materials an ideal candidate for further investigation. Further development is needed to relate macroscopically observed mechanical behavior to microscopic parameters. The diversity of weak and strong molecular integrations makes hybrid gels of various kinds a fertile area of research. In many applications, the use of hydrogels is often severely limited by their mechanical properties. For example, the poor mechanical stability of hydrogels used for cell encapsulation often leads to unintended cell release and death [29], and low toughness limits the durability of contact lenses [30]. Hydrogels of superior stiffness, toughness, stretchability and recoverability will improve the performance in these applications, and likely open up new areas of application for this class of materials.

\section{Supplementary Material}

Refer to Web version on PubMed Central for supplementary material.

\section{Acknowledgments}

The work at Harvard was supported by ARO (W911NF-09-1-0476), DARPA (W911NF-10-1-0113), and MRSEC (DMR-0820484). X.H.Z. acknowledges the support of the NSF Research Triangle MRSEC (DMR-1121107) and Haythornthwaite Research Initiation Grant. K.H.O is supported by the National Research Foundation of Korea (NRF) funded by the Ministry of Education, Science and Technology (R11-2005-065). Z.G.S. acknowledges a sabbatical leave at the Karlsruhe Institute of Technology funded by the Alexander von Humboldt Award and by Harvard University.

\section{References}

1. Lee KY, Mooney DJ. Hydrogels for tissue engineering. Chem Rev. 2001; 101:1869-1879. [PubMed: 11710233]

2. Qiu Y, Park K. Environment-sensitive hydrogels for drug delivery. Ad Drug Delivery Rev. 2001; 53:321-339.

3. Dong L, Agarwal AK, Beebe DJ, Jiang HR. Adaptive liquid microlenses activated by stimuliresponsive hydrogels. Nature. 2006; 442:551-554. [PubMed: 16885981]

4. Discher DE, Mooney DJ, Zandstra PW. Growth factors, matrices, and forces combine and control stem cells. Science. 2009; 324:1673-1677. [PubMed: 19556500]

5. Calvert P. Hydrogels for soft machines. Adv Mater. 2009; 21:743-756.

6. Okumura Y, Ito K. The polyrotaxane gel: a topological gel by figure-of-eight cross-links. Adv Mater. 2001; 13:485-487.

7. Haraguchi K, Takehisa T. Nanocomposite hydrogels: a unique organic-inorganic network structure with extraordinary mechanical, optical and swelling/de-swelling properties. Adv Mater. 2002; 14:1120-1124.

8. Lake GJ, Thomas AG. The strength of highly elastic materials. Proc R Soc A. 1967; 300:108-119.

9. Simha NK, Carlson CS, Lewis JL. Evaluation of fracture toughness of cartilage by micropenetration. J Mater Sci Mater Med. 2003; 14:631-639.

10. Lake GJ. Fatigue and fracture of elastomers. Rubber Chem Tech. 1995; 68:435-460. 
11. Gong JP, Katsuyama Y, Kurokawa T, Osada Y. Double-Network Hydrogels with Extremely High Mechanical Strength. Adv Mater. 2003; 15:1155-1158.

12. Huang T, Xu HG, Jiao KX, Zhu LP, Brown HR, Wang HL. A novel hydrogel with high mechanical strength: a macromolecular microsphere composite hydrogel. Adv Mater. 2007; 19:1622-1626.

13. Sakai T, Matsunaga T, Yamamoto Y, Ito C, Yoshida R, Suzuki S, Sasaki N, Shibayama M, Chung U. Design and Fabrication of a High-Strength Hydrogel with Ideally Homogeneous Network Structure from Tetrahedron-like Macromonomers. Macromolecules. 2008; 41:5379-5384.

14. Seitz ME, Martina D, Baumberger T, Krishnan VR, Hui C-Y, Shull KR. Fracture and large strain behavior of self-assembled triblock copolymer gels. Soft Matter. 2009; 5:447-456.

15. Lin W-C, Fan W, Marcellan A, Hourdet D, Creton C. Large Strain and Fracture Properties of Poly(dimethylacrylamide)/Silica hybrid hydrogels. Macromolecules. 2010; 43:2554-2563.

16. Wang QG, Mynar JL, Yoshida M, Lee EJ, Lee MS, Okuro K, Kinbara K, Aida T. High-watercontent mouldable hydrogels by mixing clay and a dendritic molecular binder. Nature. 2010; 464:339-343. [PubMed: 20090750]

17. Haque MA, Kurokawa T, Kamita G, Gong JP. Lamellar bilayers as reversible sacrificial bonds to toghen hydrogel: hysteresis, self-recovery, fatigue resistance, and crack blunting. Macromolecules. 2011; 44:8916-8924.

18. Tuncaboylu DC, Sari M, Oppermann W, Okay O. Tough and self-healing hydrogels formed via hydrophobic interactions. Macromolecules. 2011; 44:4997-5005.

19. Hui CY, Jagota A, Bennison SJ, Londono JD. Crack blunting and the strength of soft elastic solids. Proc R Soc Lond A. 2003; 459:1489-1516.

20. Yu QM, Tanaka Y, Furukawa H, Kurokawa T, Gong JP. Direct observation of damage zone around crack tips in double-network gels. Macromolecules. 2009; 42:3852-3855.

21. Webber RE, Creton C, Brown HR, Gong JP. Large strain hysteresis and mullinsV effect of tough double-network hydrogels. Macromolecules. 2007; 40:2919-2927.

22. Henderson KJ, Zhou TC, Otim KJ, Shull KR. Ionically cross-linked triblock copolymer hydrogels with high strength. Micromolecules. 2010; 43:6193-6201.

23. Kong HJ, Wong E, Mooney DJ. Independent control of rigidity and toughness of polymeric hydrogels. Macromolecules. 2003; 36:4582-4588.

24. Baumberger T, Ronsin O. From thermally activated to viscosity controlled fracture of biopolymer hydrogels. J Chem Phys. 2009; 130:061102. [PubMed: 19222257]

25. Evans AG. Perspective on the development of high-toughness ceramics. J Am Ceram Soc. 1990; 73:187-206.

26. Brown HR. A model of fracture of double network gels. Macromolecules. 2007; 40:3815-3818.

27. Tanaka Y. A local damage model for anomalous high toughness of double-network gels. EPL. 2007; 78:56005.

28. Jackson AP. Measurement of the fracture toughness of some contact lens hydrogels. Biomater. 1990; 11:403-407.

29. Hernandez RM, Orive G, Murua A, Pedraz JL. Microcapsules and microcarriers for in situ cell delivery. Adv Drug Deliv Rev. 2010; 62:711-730. [PubMed: 20153388]

30. Maldonado-Codina C, Efron N. Impact of manufacturing technology and material composition on the mechanical properties of hydrogel contact lenses. Ophthal Physiol Opt. 2004; 24:551-561. 

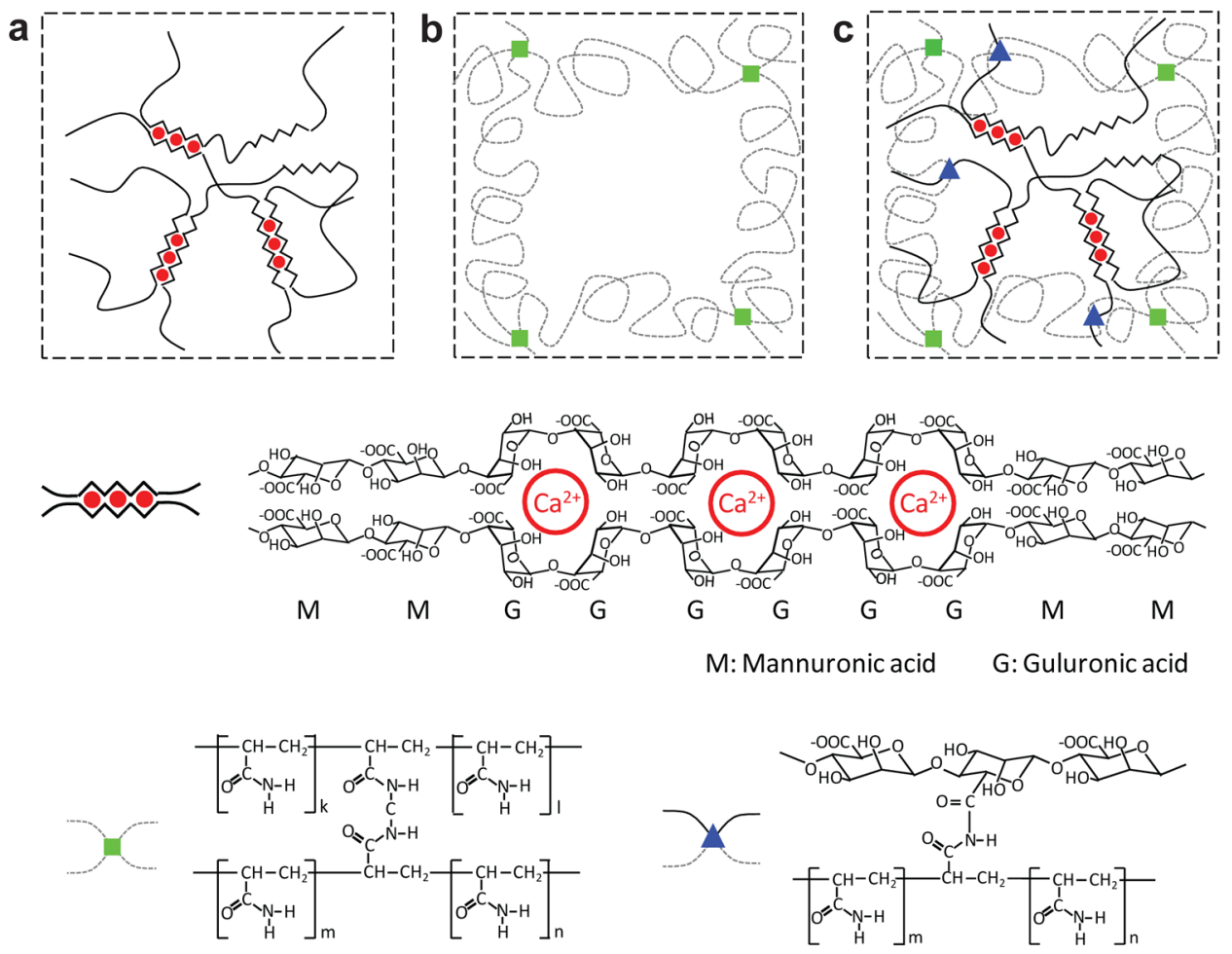

Figure 1. Schematics of three types of hydrogels

a, In an alginate gel, the $\mathrm{G}$ blocks on different polymer chains form ionic crosslinks through $\mathrm{Ca}^{2+}$. b, In a polyacrylamide gel, the polymer chains form covalent crosslinks through MBAA. c, In an alginate-polyacrylamide hybrid gel, the two types of polymer networks are intertwined. 

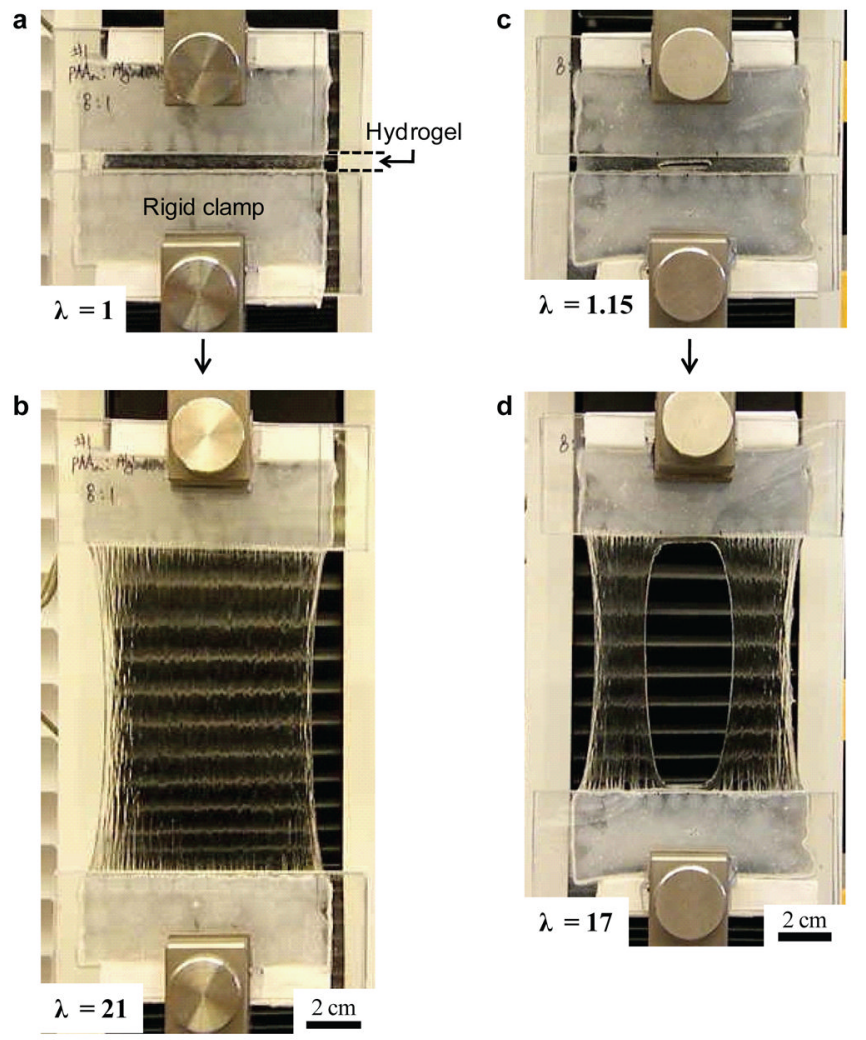

Figure 2. The hybridgel is highly stretchable and notch-insensitive

a, A strip of the undeformed gel was glued to two rigid clamps. b, The gel was stretched 21 times its initial length. The stretch $\lambda$ is defined by the distance between the two clamps when the gel is deformed divided by the distance when the gel is undeformed. c, A notch was cut into the gel by using a razor blade; a small stretch of 1.15 was used to make the notch clearly visible. $\mathbf{d}$, The gel containing the notch was stretched 17 times its initial length. The alginate-to-acrylamide ratio was 1:8. The covalent crosslinker, MBAA, was fixed at 0.0006 the weight of acrylamide. The ionic crosslinker, CaSO4, was fixed at 0.1328 the weight of alginate. 
a

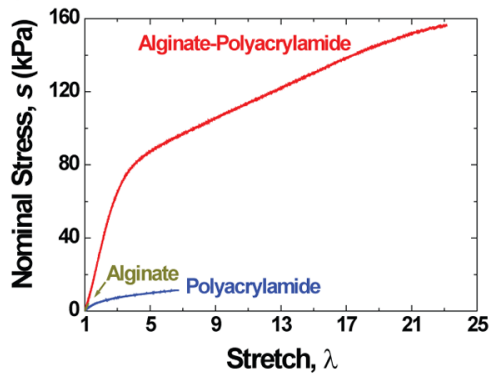

d

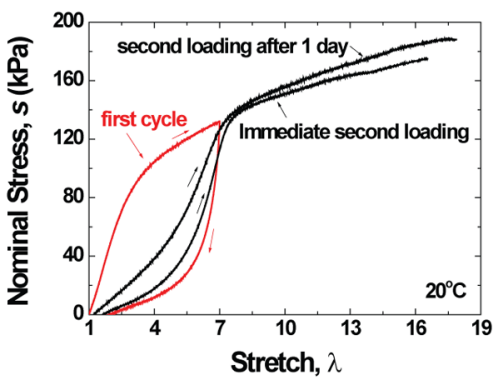

b

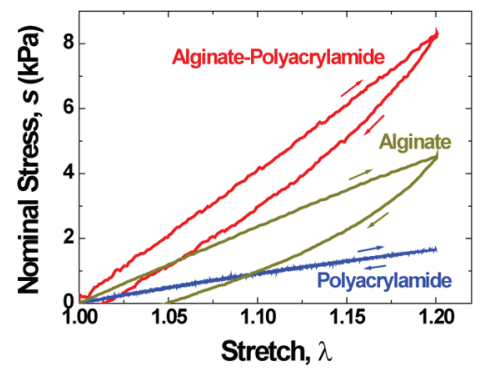

e

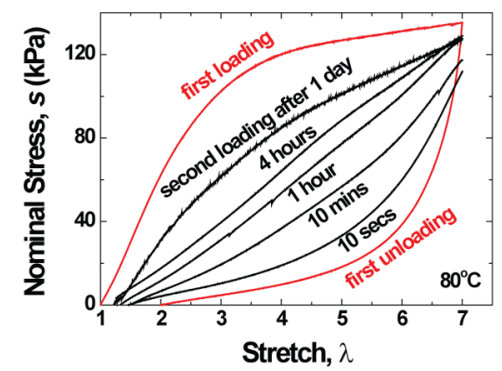

C

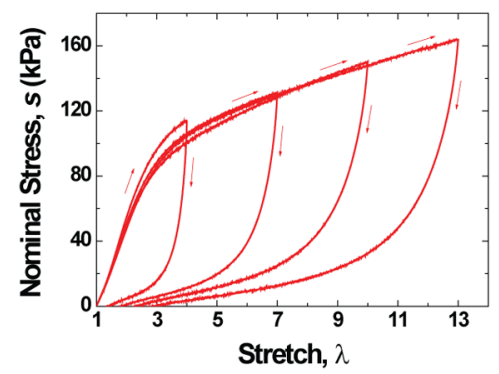

f

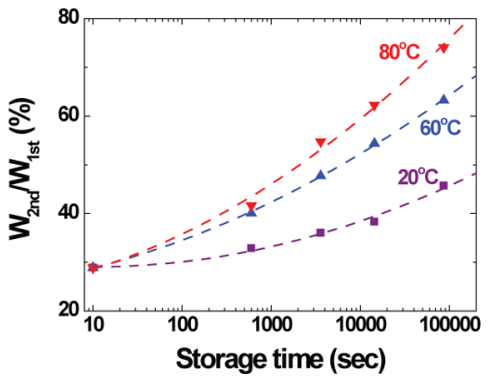

Figure 3. Mechanical tests under various conditions

a, Stress-stretch curves of the three types of gels, each stretched to rupture. The nominal stress $s$ is defined by the force applied on the deformed gel divided by the cross-sectional area of the undeformed gel. b, The gels were each loaded to a stretch of 1.2, just below the value that would rupture the alginate gel, and were then unloaded. c, Samples of the hybrid gel were subject to a cycle of loading and unloading of varying maximum stretch. d, After the first loading and unloading, one sample was reloaded immediately, and the other sample was reloaded after 1 day. e, Recovery of samples stored at $80^{\circ} \mathrm{C}$ for different durations of time. $\mathbf{f}$, The work of the second loading $W_{2 n d}$ normalized by that of the first loading $W_{1 s t}$ was measured for samples stored for different periods of time at different temperatures. The alginate-to-acrylamide ratio was 1:8 for a and $\mathrm{b}$, and was 1:6 for $\mathrm{c}-\mathrm{f}$. The covalent crosslinker, MBAA, was fixed at 0.0006 the weight of acrylamide for polyacrylamide gel and hybrid gel. The ionic crosslinker, $\mathrm{CaSO} 4$, was fixed at 0.1328 the weight of alginate for alginate gel and hybrid gel. 
a

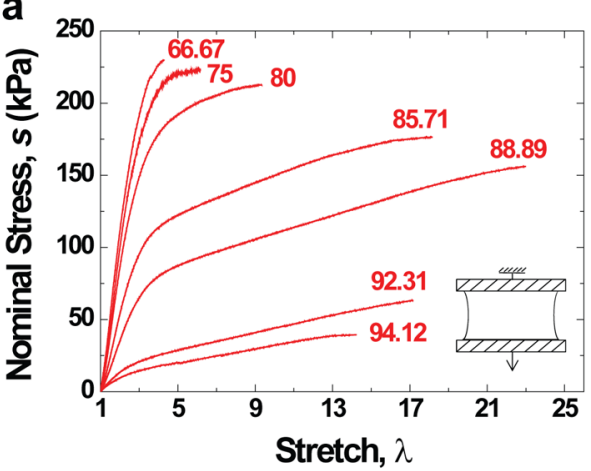

C

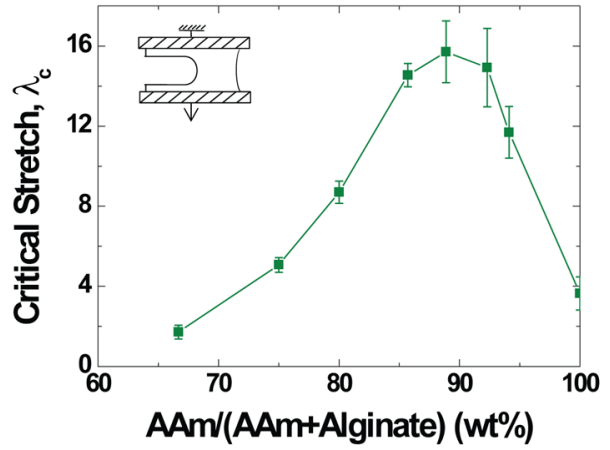

b

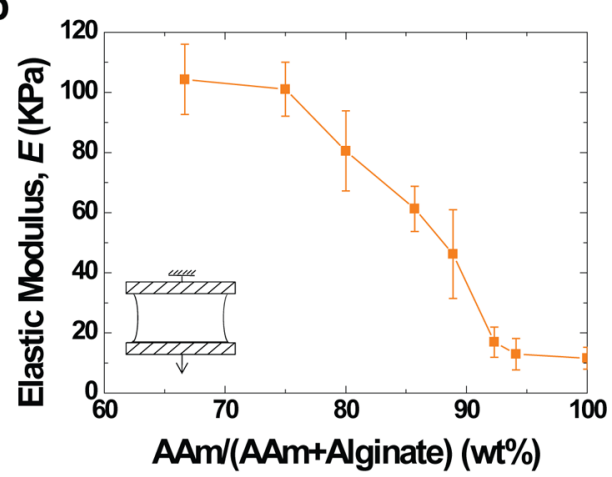

d

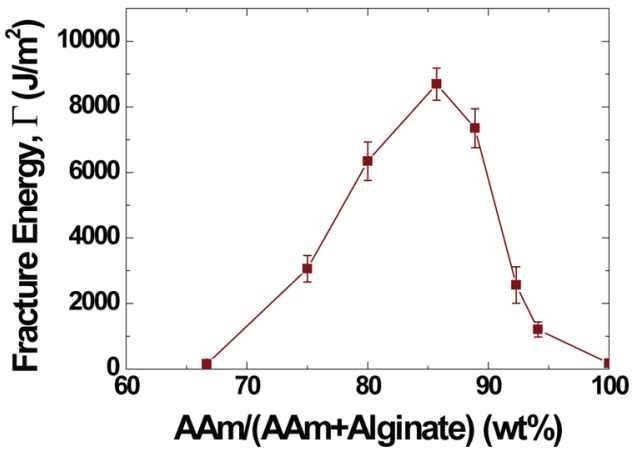

Figure 4. The composition greatly affects the behavior of the hybrid gel

a, Stress-strain curves of gels of various weight ratios of acrylamide and alginate. Each test was conducted by pulling an unnotched sample to rupture. b, Elastic moduli were calculated from stress-strain curves. c, Notched gels of various acrylamide-to-alginate ratios were pulled to rupture to measure the critical stretches. d, Fracture energy was plotted as a function of the acrylamide-to-alginate ratio. The covalent crosslinker, MBAA, was fixed at 0.0006 the weight of acrylamide. The ionic crosslinker, $\mathrm{CaSO} 4$, was fixed at 0.1328 the weight of alginate. (Error bars, S.D.; $n=4$ ) 